\title{
Trabajos Libres Ganadores XXXVIII Congreso Latinoamericano de Infectología Pediátrica
}

\author{
Winning Free Papers \\ XXXVIII Latin American Congress of Pediatric Infectology
}

Investigación en la práctica clínica

\section{PRIMER LUGAR}

\section{Absceso cerebral por Eikenella corrodens: reporte de un caso}

Valdés de la Torre GE, Martínez-Bustamante ME, MoraytaRamírez RA

Centro Médico Nacional 20 de Noviembre, ISSSTE.

Correspondencia: Gabriela Estefanía Valdés de la Torre. Eje 7 Sur Félix Cuevas, esquina con Eje 3 Poniente, Av. Coyoacán, Col. Del Valle Sur, Cd. de México. Cel: 5554692345.E-mail:stephyvaldes@gmail.com

El absceso cerebral es un reto diagnóstico y terapéutico, presenta manifestaciones clínicas y radiológicas inespecíficas. Incidencia de 0.3-1-3 casos/100,000 personas al año, 25\% en menores de 15 años. 95\% son de origen bacteriano, $80 \%$ presentan un factor de riesgo predisponente. Caso: Paciente masculino de 16 años de edad, originario de la Ciudad de México, previamente sano, inicia en febrero de 2018 con cefalea hemicraneana derecha, intermitente, intensidad 5/10, exacerbada con cambios de posición, tratamiento con AINEs, mejoría parcial. Persiste con sintomatología ocho semanas agregándose anhedonia. El 9.04.18 aumentó de intensidad de cefalea 8/10, dolor retroocular, náuseas y vómito. El 10.04.18 se hospitaliza por datos de hipertensión endocra-

Financiamiento: Ninguno.

Conflicto de intereses: Ninguno.

Derecho a la privacidad y consentimiento

informado: Los autores declaran que en este

artículo no aparecen datos personales del paciente.

https://dx.doi.org/10.35366/94421

Rev Latin Infect Pediatr 2020; 33 (2): 97-106 neana. EF: FC 72 Ipm, Fr 18 rpm, TA 100/60, To 36 ${ }^{\circ} \mathrm{C}$; consciente, Glasgow 15 , reflejo pupila izquierda disminuido, pares craneales sin alteraciones, Kernig y Brudzinski negativos, marcha sin alteraciones, oídos sin alteraciones, amígdalas hipertróficas III, crípticas. $\mathrm{Hg} 15.3 \mathrm{~g} / \mathrm{dL}$, Hto $45 \%$, leucos $10,200 \mathrm{~mm}^{3}$, neutros $80 \%$, linfocitos $15 \%$, plaquetas 301,000 , PCT 0.45 $\mathrm{ng} / \mathrm{mL}$, PCR $30 \mathrm{mg} / \mathrm{L}$. RM cráneo con gadolinio, absceso cerebral en fase de cápsula tardía (5.7 x 3.6 $\mathrm{cm}$ ), frontoparietal derecho. Refiere realización de endodoncia un mes previo al inicio de sintomatología. Inicia manejo empírico ceftriaxona/vancomicina/ metronidazol, drenaje por estereotaxia con cultivo; Eikenella corrodens. Completa tratamiento intravenoso cuatro semanas; ceftriaxona + metronidazol y vía oral dos semanas; ampicilina/sulbactam. Paciente con adecuada evolución clínica y radiológica. Discusión: Los abscesos cerebrales son una urgencia neurológica grave, tiempo medio de diagnóstico de ocho días. Se presenta tríada; cefalea, fiebre y focalización (20-30\%), hemocultivos $+28 \%$. Diagnóstico de elección por imagen. Cultivos positivos $68 \%$. En pacientes sanos tienen dos orígenes principales: dentales y sinusales. En este caso se trata de un origen odontogénico. Se debe sospechar origen de patología para identificar posibles microorganismos, Streptococcus $60-70 \%$, anaerobios $20-40 \%$, enterobacterias $20-30 \%$, polimicrobianos 23-30\%, Staphylococcus aureus $10-15 \%$. Tratamiento con drenaje y antibioticoterapia intravenosa por 4-6 semanas. Eikenella corrodens, bacteria del grupo HACEK, bacilo Gram negativo, anaerobio facultativo. Biota de boca, tracto digestivo y urinario. Frecuente como patógeno en periodontitis, infecciones de heridas contaminadas por secreciones orales, SNC, celulitis orbitaria, absceso periamigdalino, infecciones abdominales y endocarditis. Sensible a piperacilina, cefalosporinas de segunda-tercera 
generación, carbapenémicos y fluoroquinolonas. Algunas cepas producen B-lactamasas.

\section{SEGUNDO LUGAR}

Manifestaciones extraintestinales de infecciones por Campylobacterales: reporte de tres casos y revisión de la literatura

Cristerna-Tarrasa GH, Sarmiento-Aguilar A, AguilarGómez NE

Instituto Nacional de Pediatría.

Correspondencia: Av. Insurgentes 3700-C, Col. Insurgentes Cuicuilco, 04530, Coyoacán, México. Teléfono: 10840900.E-mail:ghct.inp@gmail.com

Objetivo: Concientizar al personal de la salud acerca de la importancia de sospechar complicaciones extraintestinales de la gastroenteritis en pacientes inmunocomprometidos. Descripción del caso: Caso 1: paciente masculino de 16 años con agammaglobulinemia ligada al X (ALX), presenta siete días de evolución con dolor, rubor local y edema en pierna derecha, se evidencia en sangre leucocitosis con predominio de neutrófilos y se concluye celulitis. Después de tres meses de evolución tórpida con múltiples antibióticos y por último esteroide, bajo la sospecha de etiología autoinmune, se tipifica Helicobacter bilis en biopsia de piel. Caso 2: paciente masculino de ocho años con meduloblastoma clásico, muestra evacuaciones disminuidas en consistencia de aproximadamente tres semanas de evolución, sin moco ni sangre, asociado a vómito e intolerancia a la vía oral. Se realiza panel gastrointestinal y búsqueda de $C$. difficile con resultados negativos. Al desarrollar fiebre asociada con datos de respuesta inflamatoria sistémica se toma hemocultivo que reporta Campylobacter jejuni. Caso 3: masculino de 10 años con ALX, acude a urgencias por ictericia generalizada y coluria, vómito de contenido gastroalimentario y evacuaciones disminuidas de consistencia, se inicia cefotaxima empírica. Presenta evolución tórpida, recibe dos esquemas de antibióticos de amplio espectro sin mejoría, 20 días después se aísla $C$. jejuni en líquido peritoneal. Discusión: Las especies de los géneros Campylobacter y Helicobacter, como parte de los campylobacterales, comúnmente provocan infecciones gastrointestinales benignas y autolimitadas en la población general. Las complicaciones extraintestinales son poco frecuentes; sin embargo, se reportan más casos pediátricos con enfermedades crónicas e inmunosupresión en los que es importante sospechar, ante un cuadro de gastroenteritis aguda, la posibilidad de traslocación bacteriana, diseminación hematógena y complicaciones extraintestinales.

\section{TERCER LUGAR}

Endoftalmitis endógena secundaria a neuroinfección, una entidad pediátrica rara

Lozano-Vázquez LE, Espinosa-Sotero MC Hospital General de México «Dr. Eduardo Liceaga».

Correspondencia: Dr. Balmis Núm. 148, Col. Doctores, 06720, Alcaldía Cuauhtémoc, Ciudad de México. Teléfono: 8117114268. E-mail: leliel.lozano@gmail.com

Introducción: La endoftalmitis endógena o metastásica resulta de la diseminación hematógena de un microorganismo que atraviesa la barrera hematoocular desde un foco primario extraocular. La incidencia es entre 2 y $10 \%$ de todos los casos de endoftalmitis. Objetivo: Describir el caso de un paciente con endoftalmitis endógena con foco primario en sistema nervioso central. Presentación de caso: Paciente masculino de 10 meses de edad, sin antecedentes de importancia para padecimiento actual, esquema de vacunación completo para la edad que inicia el día 15.12 .18 con irritabilidad y fiebre de 39 grados, acude a valoración con múltiples médicos quienes dan diagnóstico de gastroenteritis aguda e infección de vías respiratorias, manejado con antipiréticos y antibióticos como amoxicilina con ácido clavulánico y amikacina. El día 18.12.18 la madre nota opacidad en ojo izquierdo, por lo que acude nuevamente a valoración con médico, quien diagnostica uveítis aguda y se envía a nuestro hospital. Al acudir al servicio de oftalmología para valoración, presenta episodio de movimientos anormales, por lo que es enviado a urgencias pediatría. A su ingreso se encuentra irritable, con hipopión en ojo izquierdo, pupila izquierda arrefléctica, con disminución de movimientos de hemicuerpo derecho, poco reactivo a estímulos, pulsos presentes, sincrónicos, con llenado capilar inmediato. Durante estancia en sala de observación persiste con eventos de crisis convulsivas complejas con duración mayor de dos minutos sin recuperar estado de consciencia entre episodios, por lo que se ingresa a UTIP para manejo de estatus epiléptico. Se inició manejo antibiótico con 
Rev Latin Infect Pediatr 2020; 33 (2): 97-106

vancomicina $60 \mathrm{mg} / \mathrm{kg} /$ día y ceftazidima $150 \mathrm{mg} / \mathrm{kg} /$ día, valorado por el servicio de oftalmología, quien diagnostica endoftalmitis endógena que amerita tratamiento antibiótico intravítreo; sin embargo, por condiciones hemodinámicas no se administra tratamiento. El 23.12.18 presenta bradicardia con ausencia de pulsos periféricos, por lo que se inician maniobras de reanimación avanzada, sin regreso a circulación espontánea. Padres no autorizan autopsia. Conclusiones: Como se menciona en la literatura, el diagnóstico y tratamiento de endoftalmitis endógena se retrasa debido a la ausencia inicial de síntomas que nos pueden orientar al foco de diseminación.

\section{TERCER LUGAR}

Manejo temprano de encefalitis por anticuerpos anti-GAD65 en paciente pediátrico del Hospital San Javier

Contreras MR, Márquez-Palacios R, Sánchez-Rodríguez A Hospital San Javier.

Correspondencia: Pablo Casals Núm. 640, Col. Prados Providencia, 44670, Guadalajara, Guadalajara. Teléfono: 3310963411.E-mail:mrcpcharito@hotmail.com

Objetivo: Presentar caso clínico de una paciente de 10 años de edad en quien se utilizó el puntaje APE para el manejo temprano de encefalitis por anticuerpos anti-GAD65. Caso clínico: Paciente femenino de 10 años, con antecedente de infección de vías respiratorias altas una semana previa al padecimiento, en tratamiento con ácido acetil salicílico, benzonatato y pramigel. Por medio de interrogatorio indirecto, familiar comenta que la noche previa tuvo episodios de déjà vu y somnolencia, por la madrugada se cae de la cama, al parecer secundario a evento convulsivo y la encuentran con alteración de la conciencia, lenguaje incoherente e hipotonía generalizada. A su ingreso presenta evento de supraversión de la mirada, movimientos faciales y tonicoclónicos generalizados con una duración menor de cinco minutos y postictal con somnolencia sin recuperación de la conciencia, por lo que se aplica intubación endotraqueal. Se inicia abordaje para síndrome encefálico, se realiza TAC, RMN y punción lumbar que revelan sólo lactato elevado. Los estudios complementarios para infección fueron negativos, se mantiene intubada por tres días y posteriormente, a la exploración física, la paciente se muestra indiferente al medio, no interactúa, no obedece comandos, con ptosis derecha, tono aumentado en cuatro extremidades, predominio de lado derecho, hiperreflexia universal, Babinski derecho, atrapamiento de reflejo aquíleo derecho, sin movimientos intencionados y sin signos meníngeos ni atávicos. Posteriormente muestra mejoría clínica, recuperando el estado de alerta y el habla en frases cortas; sin embargo, al día siguiente continúa con crisis epilépticas de tipo focal con bilateralización, deterioro neurológico, fiebre de bajo grado, variaciones en tensión arterial y frecuencia respiratoria, temblor en reposo, así como cambios conductuales como indiferencia al medio y agitación. Por los datos clínicos y temporales que presentó la paciente, así como resultados de laboratorio negativos para infección, se sospecha de encefalitis autoinmune antineuronal, se calcula APE score de $13 / 15$ y se decide iniciar con tratamiento a base de inmunoglobulina intravenosa $2 \mathrm{~g} / \mathrm{kg}$ dosis total dividida en cuatro dosis, complementario a manejo antiepiléptico con levetiracetam y ácido valproico. Existe mejoría posterior a la administración de inmunoglobulina, se encuentra alerta, orientada en lugar y persona, obedece comandos sencillos, con lenguaje coherente, en ocasiones soliloquios, dificultad para cálculo, no entiende pensamiento abstracto, ptosis derecha, trofismo adecuado, tono aumentado, predominio derecho proporcionado, hiperreflexia universal, sin Babinski, logra marcha, torpe, hemiparética, con temblor fino de reposo. Cuatro semanas posteriores al inicio de la terapia la paciente sólo continuó con cambios en el comportamiento. Con resultados de anticuerpos positivos para anti-GAD65. Estudios de laboratorio y gabinete: 26-07: biometría hemática: hemoglobina $15.45 \mathrm{~g} / \mathrm{dL}$, hematocrito $48.35 \%$, leucocitos $16.55 / \mu \mathrm{L}$, neutrófilos 13.57/ $\mu \mathrm{L}$, linfocitos $1.65 / \mu \mathrm{L}$. 26-07: toxicológico negativo. 26-07: química sanguínea: glucosa 112 $\mathrm{mg} / \mathrm{dL}$, ácido úrico $11.9 \mathrm{mg} / \mathrm{dL}$, AST $78 \mathrm{U} / \mathrm{L}, \mathrm{ALT}$ $51 \mathrm{U} / \mathrm{L}, \mathrm{FA} 183 \mathrm{U} / \mathrm{L}, \mathrm{GGT} 113 \mathrm{U} / \mathrm{L}, \mathrm{DHL} 798 \mathrm{U} / \mathrm{L}$. 26-07: citoquímico de LCR: transparente, incoloro, glucosa $90 \mathrm{mg} / \mathrm{dL}$ (112 sérica), proteínas totales 27 $\mathrm{mg} / \mathrm{dL}$, cloro $134 \mathrm{mmol} / \mathrm{L}$, no se observan células blancas, células rojas, VDRL negativo, tinción Gram negativa, lactato $2 \mathrm{mmol} / \mathrm{L}$. 28-07: hemocultivo negativo. 31-07: hemoglobina $10.72 \mathrm{~g} / \mathrm{dL}$, hematocrito $34.3 \%$, leucocitos $10.6 / \mu \mathrm{L}$, neutrófilos $7.38 / \mu \mathrm{L}$, linfocitos 1.99/ $\mu \mathrm{L}$. 31-07: examen general de orina: células epiteliales abundantes, bacterias escasas, 
eritrocitos 4 a 5 por campo, leucocitos 1 a 2 por campo, nitritos negativos. 31-07: procalcitonina 2.4, PCR 13, electrolitos séricos normales. 31-07: PCR múltiple para infección de virus SNC en LCR (VHS1,2, VVZ, ENT, VEB, CMV, LCMV): negativo. PCR para M. tuberculosis: negativo. 04-08: anticuerpos anti-Mycoplasma pneumoniae: IgG 23.7, IgM negativos. 09-08: anticuerpos antinucleares: 1:160. ANCA: p-ANCA: 9.6, c-ANCA 8. Complemento: C3 162, C4 20. 02-09: anticuerpos anti-receptor NMDA IgG: negativos. Anticuerpos antiglutamato descarboxilasa 65:136 IU/mL. Electroencefalograma: datos de disfunción cortical generalizada, no se observa actividad irritativa ni eliptiforme. Seguimiento: pronóstico: bueno para la vida, reservado para la función. Discusión: De acuerdo con la literatura, la encefalitis de origen autoinmune es una entidad poco diagnosticada, ésta se clasifica por el origen de los antígenos en la célula, ya sea en la superficie celular o en el citoplasma. En las de origen citoplásmico, en particular la encefalitis por anticuerpos antiácido glutámico descarboxilasa (GAD65), representa una etiología poco común, con un gran porcentaje de casos refractarios y con mal pronóstico. Estos anticuerpos se encuentran positivos en aproximadamente $17 \%$ de los casos de encefalitis límbica. Se presenta sobre todo en mujeres jóvenes, hay muy pocos casos reportados en pacientes pediátricos. El antígeno GAD65 es una enzima intracitoplásmica, la cual convierte glutamato en GABA, se encuentra alterada en enfermedades como diabetes mellitus tipo 1, ataxia cerebelosa, autoinmunidad poliendócrina, epilepsia, entre otros. La manifestación clínica que mostrará el paciente dependerá del sitio de unión del anticuerpo, en el caso de la encefalitis autoinmune, la unión de los anticuerpos es principalmente en la región carboxiterminal de la molécula. La encefalitis límbica representa el cuadro clínico clásico, comienza con crisis convulsivas refractarias, cambios en el comportamiento y disminución de la cognición. Para hacer el diagnóstico clínico existen herramientas como el APE score, el cual evalúa la probabilidad de tener anticuerpos positivos al presentar un cuadro de crisis convulsivas de etiología desconocida. Un valor igual o mayor de cuatro puntos tiene una sensibilidad y especificidad de 82 y $82.6 \%$ respectivamente. Este puntaje puede utilizarse como herramienta en pacientes para decidir iniciar terapia empírica y hacer la toma de anticuerpos. Entre los estudios auxiliares, la resonancia magnética en algunos casos muestra un reforzamiento en el lóbulo temporal en T2; sin embargo, en algunos casos, el estudio se encuentra dentro de parámetros normales, por lo que es recomendable repetirlo en cuatro meses. En el EEG no se muestran datos específicos; sin embargo, la mayoría de los pacientes tienen descargas ictales o interictales en el lóbulo temporal. El diagnóstico definitivo se hace por la detección de anticuerpos anti-GAD65 en LCR o plasma, se puede utilizar ELISA, inmunohistoquímica y posteriormente Western blot y radioinmunoanálisis para confirmar. La terapia de primera línea consiste en metilprednisolona en pulsos por cinco días seguida de prednisona oral por un mes e inmunoglobulina intravenosa por cuatro o cinco días y recambio con plasma. Esto ha demostrado reducir el número de crisis convulsivas y mejorar los cambios conductuales y la cognición. En casos recidivantes, el tratamiento de segunda línea es a base de rituximab, ciclofosfamida o micofenolato de mofetilo. Es importante iniciar el tratamiento de inmediato para evitar secuelas como déficit cognitivo, crisis convulsivas refractarias, cambios en el comportamiento y disautonomías, ya que en ocasiones es letal para el paciente.

Referencias

1. Achour NB, Younes TB, Rebai I, Ahmed MB, Kraoua I, Youssef-Turki IB. Severe dysautonomia as a main feature of anti-GAD encephalitis: report of a paediatric case and literature review. Eur J Paediatr Neurol. 2018; 22 (3): 548-551.

2. Dubey D, Alqallaf A, Hays R, Freeman M, Chen K, Ding K et al. Neurological autoantibody prevalence in epilepsy of unknown etiology. JAMA Neurol. 2017; 74 (4): 397-402.

3. Daif A, Lukas RV, Issa NP, Javed A, VanHaerents S, Reder AT et al. Antiglutamic acid decarboxylase 65 (GAD65) antibody-associated epilepsy. Epilepsy Behav. 2018; 80: 331-336.

Infecciones Asociadas en Atención a la Salud

\section{PRIMER LUGAR}

Erradicación de infecciones por

microorganismos productores de BLEE con uso de coberturas de cobre y desinfección UV

Casillas-Casillas MC, De la Rosa-Zamboni D, JiménezJuárez R, Velázquez-Guadarrama $N$, Villegas-Silva $R$, Minero-Hibert MA, Moreno-Miranda R, Rosas-Mateos I, Jiménez $\mathrm{V}$, Franco I

Instituto Nacional de Salud Hospital Infantil de México «Federico Gómez». 
Rev Latin Infect Pediatr 2020; 33 (2): 97-106

Correspondencia: Dr. Márquez Núm. 162, Col. Doctores, 06720, Cuauhtémoc, Ciudad de México. Teléfono: 52289917, ext.2331.E-mail:medicit_casillas@hotmail.com

Objetivo: Analizar el impacto de estrategias para la reducción de CLABSI y ESBL en la Unidad de Cuidados Intensivos Neonatales del Hospital Infantil de México «Federico Gómez» mediante implementación de superficies «auto-desinfectantes» a base de un recubrimiento de cobre y desinfección con UV-C. Introducción: Las bacterias BLEE son causantes de alta mortalidad y morbilidad en todo el mundo. Unidades de cuidados intensivos neonatales (UCIN) reportan múltiples brotes donde la letalidad es aún mayor (40\%). Se ha descrito la diseminación de BLEE entre pacientes tanto por trabajadores de salud como por reservorios ambientales. En nuestra UCIN la prevalencia de BLEE se había mantenido en ascenso desde los últimos tres años. Como medida de control implementamos bundles vs. CLABSI, limpieza exhaustiva con UV-C seguida de la colocación de placas de cobre. En el presente estudio analizamos el impacto de tales maniobras en $\mathrm{HCAl}$, aislamientos clínicos $\mathrm{BLEE}+\mathrm{y}$ genes de resistencia. Material y métodos: Estudio antes-después para evaluar el impacto de la colocación de placas de desinfección con UV seguida de colocación de $\mathrm{Cu}+$ en superficies de alto contacto (barandales de incubadoras, mesas de trabajo, manijas). Se midió prevalencia de HCAI en UCIN, HCAI con ESLB+ y ESBL en todo aislamiento clínico. Los aislamientos ESBL+ fueron comprobados por reacciones en cadena de la polimerasa (PCR) para detectar la presencia de genes codificantes de ESBL, con iniciadores específicos para CTX-M, TEM, SHV, GES, VEB, PER, KPC, VIM e IMP (diseñados por Dallene 2010), para las $\beta$-lactamasas tipo AmpC (ACC, ACT, DHA, CMY, FOX, LAT, MIR y MOX), las metalo-carbapenemasas (GIM, NDM, SIM y SPM), las serin-carbapenemasas (IMI, SME y NMC-A) y las OXA $\beta$-lactamasas (grupos OXA 23, 24, 48, $1,2,51,4$ y 58 ) metodología sugerida por Voets (2011). Resultados: Se obtuvo una N de 316 recién nacidos, se siguieron 17,362 pacientes en 18 meses. Se observó una disminución de la tasa de HCAI de 10.4 a 5.39/1,000 días paciente después del conjunto de medidas de control. Los aislamientos clínicos estudiados previamente mostraron una tendencia a incrementar el porcentaje de genes de resistencia, la cual disminuyó tras medidas de control y al igual que las HCAI BLEE+ disminuyeron desde el uso de desinfección UV y Cu+. No obtuvimos aislamientos
$\mathrm{BLEE}+\mathrm{y}$ genes de resistencia en seguimiento posterior a seis meses. Conclusiones: Presentamos por primera vez un reporte de eliminación de transmisión de enterobacterias BLEE + y genes de resistencia en una UCIN mediante la utilización de desinfección UV seguida de placas de $\mathrm{Cu}$ en superficies de alto contacto. Este hallazgo sugiere un rol predominantemente ambiental en la transmisión de enterobacterias con posibilidad de su eliminación a través de estas estrategias.

\section{SEGUNDO LUGAR}

Epidemiología, factores de riesgo y

susceptibilidad de infecciones fúngicas invasivas en niños con cáncer del norte de México

Riojas-Hernández MP, Vaquera-Aparicio DN, Mares-Gil JE, Castillo-Bejarano JI, Mascareñas de los Santos A Hospital Universitario «Dr. José Eleuterio González».

Correspondencia: Dr. José Eleuterio González (Gonzalitos) S/N, Col. Mitras Centro, 64460, Monterrey, N.L. Teléfono: 8115194201.E-mail: patyriojas90@gmail.com

Introducción: Las infecciones fúngicas invasivas más documentadas a nivel mundial son Candida spp. y Aspergillus spp. En México desconocemos la epidemiología en pacientes pediátricos con cáncer. Objetivos: Describir epidemiología, factores de riesgo y susceptibilidad de fungemias en niños con cáncer. Material y métodos: Estudio descriptivo, retrospectivo. Incluimos pacientes pediátricos con cáncer de noviembre 2017 a septiembre 2019, del Hospital Universitario «Dr. José Eleuterio González», Monterrey, N.L. Datos recopilados de expediente clínico y laboratorio de micología. El diagnóstico de IFI se realizó de acuerdo con los criterios establecidos por la EORTC/ MSG. Resultados: 50 pacientes (de uno a 16) años con diagnóstico de neoplasia hematooncológica ingresados al departamento de pediatría. Diagnóstico de IFI probada en ocho pacientes (n: 50 , IFI: 9, $18 \%$ ), un paciente IFI probable. Predominó sexo masculino (77.7\%). La neoplasia predominante fue LLA células $B(n=5,55.5 \%)$; todos los pacientes con LLA en fase de inducción a la remisión de QT. Factores de riesgo identificados: antibioticoterapia amplio espectro en $100 \%$, cinco portadores de CVC; seis en neutropenia severa, ocho recibiendo quimioterapia y de ellos seis en fase de inducción a la remisión; cinco con infección bacteria- 
na concomitante. Diagnóstico de candidemia en un paciente; tres fusariosis invasiva (Fusarium spp. en biopsia y sangre). Uno con mucormicosis rinosinusal y palatina (Apophysomyces spp. en biopsia); tres aspergilosis pulmonares probadas (dos cultivos de LBA y una biopsia) y una probable (galactomanano en LBA). Todos iniciaron tratamiento empírico con anfotericina $B$ liposomal y se dirigió tratamiento de acuerdo con susceptibilidad. Mortalidad 11\%. Ética: no se requirió consentimiento informado. Conclusiones: Fusariosis fue la fungemia más frecuente. Antibióticos de amplio espectro, neutropenia grave y cursar fase de inducción fueron los factores de riesgo encontrados.

\section{TERCER LUGAR}

Brote de Acinetobacter baumannii/calcoaceticus complex en la unidad de cuidados intensivos de un hospital pediátrico de tercer nivel

Cristerna-Tarrasa GH, Aquino-Andrade A, Mérida-Vieyra $\mathrm{J}$, De Colsa-Ranero A, Arias de la Garza E Instituto Nacional de Pediatría.

Correspondencia: Av. Insurgentes 3700-C, Col. Insurgentes Cuicuilco, 04530, Coyoacán, México. Teléfono: 10840900.E-mail: ghct.inp@gmail.com

Objetivo: Analizar un brote de Acinetobacter baumannii/calcoaceticus complex. Material y métodos: Se analizó un brote de $A$. baumannii en la Unidad de Terapia Intensiva (UTI) del Instituto Nacional de Pediatría. Se realizaron cultivos de los ambientes. La identidad y perfil de susceptibilidad de los aislamientos se obtuvo de un equipo semiautomatizado. Se determinó la relación clonal con electroforesis en gel de campos pulsados (PFGE). Se registraron las medidas de contención y control del brote. Resultados: Se detectaron cuatro aislamientos de $A$. baumannii del 30 de abril al 06 de junio de 2019 en las camas 269, 270, 271 y 272 de la UTI. Los cuatro casos cursaron con estancia hospitalaria > dos semanas, tratamiento prolongado con antimicrobianos de amplio espectro. Tres de los cuatro casos presentaron inmunocompromiso y el cuarto tenía enfermedad neurológica. El foco del caso índice fue bacteriemia y los siguientes tres casos, neumonía asociada a ventilador. Los aislamientos sólo fueron susceptibles a colistina. Se indicó tratamiento intravenoso e inhalado con colistina a tres pacientes, dos resolvieron la infección, uno falleció por neumonía; el cuarto paciente no recibió tratamiento por encontrarse en fase terminal. La PFGE reveló que los cuatro aislamientos de los pacientes y el obtenido de la toma de oxígeno de la cama 273 mostraron que tenían el mismo origen clonal. Se realizó limpieza exhaustiva y desinfección con luz UV, posterior a esto se detuvo el brote. Se continúa con la vigilancia estrecha hasta cumplir cinco meses del último caso. Conclusiones: Las infecciones hospitalarias por microorganismos MDR han ido en aumento. El detectar rápidamente el origen del brote con la consecuente identificación clonal por biología molecular permitió establecer de inmediato el control e iniciar un seguimiento estrecho del brote. Con base en los resultados, se deben implementar estrategias tanto específicas y universales para prevenir recurrencias y disminuir morbimortalidad.

\section{Investigación Básica}

\section{PRIMER LUGAR}

Estandarización de un método de PCR rápido (fast PCR) para determinar genes de producción de biofilm icaA e icaD en cepas nosocomiales de staphylococci

Cabrera-Contreras R, Morelos-Ramírez R, MeléndezHerrada E

Departamento de Salud Pública, Facultad de Medicina, UNAM.

Correspondencia: Av. Universidad 3000, Col. Copilco Universidad, 04510, Coyoacán, CDMX. Teléfono: 56232248.E-mail: cabreracontrerasr@yahoo.com.mx

Objetivo: Estandarización de un fast PCR para la identificación de los genes ica $A$ e icaD implicados en la formación de biofilm en cepas clínicas de staphylococci. Tipo y diseño del estudio: Estudio observacional descriptivo en pacientes pediátricos y adultos, con infecciones nosocomiales (IN) de cepas de $S$. aureus (SA) y $S$. epidermidis (SE), con previa identificación fenotípica y molecular con PCR clásico. Material y métodos: Se estudiaron 23 cepas para la estandarización, distribuidas en 19 de SA y cuatro de SE (Instituto Nacional de Cardiología Ignacio Chávez, SSA; Hospital General «Dr. Darío Fernández Fierro» y CMN 20 Noviembre, ISSSTE). Se estandarizó con Biorad C-1000 el ADN purificado por digestión con 
Rev Latin Infect Pediatr 2020; 33 (2): 97-106

lisozima y lisostafina. Las condiciones fueron, hot lid: $105^{\circ} \mathrm{C}$. (1.), temp. $95^{\circ} \mathrm{C}, 120$ seg. (2.), temp. 95 ${ }^{\circ} \mathrm{C}, 5$ seg. (3.), temp. $58.1^{\circ} \mathrm{C}, 10$ seg. (4.), repetir (2) 34 veces. Tubos con volumen final de $10 \mu \mathrm{L}$, con reactivo SSO Advanced Universal Probes Supermix (Biorad). Duración 30 minutos. En gel se observa un amplicon de $125 \mathrm{pb}$ en icaA y $296 \mathrm{pb}$ en icaD. Se emplearon iniciadores: icaA.F. TCATATTTGTAAGTTGGTATCC icaA.R. TCTGTCTGGGCTTGACCAT icaD.F. ATGGTCAAGCCCAGACAGAG icaD.R. CTCCCTCTCTGCCATTTTTG. Resultados:

\begin{tabular}{ll} 
Hospital & Resultados \\
\hline ISSSTE & icaA+ $15 / 15$; icaD+ $15 / 15$ (S. aureus) \\
ISSSTE & icaA+ $3 / 3$; icaD $+3 / 3$ (S. aureus) \\
ISSSTE & icaA- 4/3 icaD- $4 / 3$ (S. epidermidis) \\
ISSSTE & icaA- $1 / 3$ icaD- $1 / 3$ (S. haemolyticus)
\end{tabular}

$(+)=$ Presencia del gen, $(-)=$ ausencia del gen .

Conclusiones: La detección de cepas clínicas de staphylococci por los métodos fenotípicos convencionales y el PCR clásico generan resultados adecuados, pero con una gran inversión de tiempo. Encontramos una alta correlación entre los resultados del PCR rápido (fast) con los ensayos fenotípicos y de PCR clásico, en las cepas de $S$. aureus de los hospitales CMN 20 Noviembre y Hospital «Lic. Adolfo López Mateos». Para los resultados de las cepas del hospital en el D.F. se incluyeron tres cepas de $S$. aureus, cuatro cepas de $S$. epidermidis y una cepa de $S$. haemolyticus. El tiempo de amplificación de los genes estudiados en el protocolo de fast PCR propuesto en este trabajo es de 30 minutos en comparación con el protocolo clásico, que es de 80 minutos, observándose una reducción importante de tiempo de casi una hora para la amplificación de los genes icaA e icaD. La ventaja del PCR fast es que permite la detección de estos genes, con la misma precisión que el PCR clásico, pero de manera más rápida. Este método permite la generación rápida de datos útiles para el diagnóstico y la epidemiología de las IN.

\section{SEGUNDO LUGAR}

Enolasa recombinante de Haemophilus influenzae potencial inmunógeno para el desarrollo de una vacuna
Osorio-Aguilar Y, ${ }^{*}$ Carabarín-Lima A, ${ }^{*, \neq}$ González-Vázquez MC, ${ }^{*}$ Martínez-Laguna $Y,{ }^{*}$ Lozano-Zaraín P, ${ }^{*}$ RochaGracia $\mathrm{RC}^{*}$

* Postgrado en Microbiología, Centro de Investigaciones en Ciencias Microbiológicas. ₹ Licenciatura en Biotecnología, Instituto de Ciencias, Benemérita Universidad Autónoma de Puebla. Puebla, Pue.

Correspondencia: Instituto de Ciencias, Edificio IC11. Ciudad Universitaria, San Claudio S/N y 24 Sur, Col. San Manuel, 72570, Puebla, México. Teléfono: 01(222)2295500, ext. 2546. E-mail: yesenia.osorio. aguilar@gmail.com

Introducción: $H$. influenzae es un bacteria Gram negativa causante de enfermedades pediátricas invasivas como meningitis, epiglotitis, neumonía, artritis séptica, pericarditis, celulitis, bacteriemia $(H$. influenzae serotipo b). Las cepas de $H$. influenzae no tipificable (HNT) están asociadas con infecciones localizadas como otitis, conjuntivitis, sinusitis, bronquitis, neumonía; también pueden ocasionar enfermedad invasiva como meningitis y sepsis en hospederos inmunocomprometidos. Objetivo: Determinar la inmunogenicidad de la enolasa recombinante de $\mathrm{H}$. influenzae $(\mathrm{rEnoHi})$ en un modelo murino. Material y métodos: Ratones hembra BALB/C de 20 días de edad, en un inicio se sangraron para obtener el suero preinmune. La enolasa recombinante se purificó a homogeneidad. Posteriormente se realizó la inmunización con $10 \mu \mathrm{g}$ de rEnoHi emulsificada 1:1 con adyuvante completo de Freund vía IP, seguida de tres refuerzos cada siete días con la misma concentración de proteína, pero con adyuvante incompleto de Freund, como control negativo se utilizó SSI. AI término del esquema de inmunización los ratones se sangraron por punción cardiaca para obtener el suero hiperinmune. Posteriormente se determinó el título e isotipo de anticuerpos policlonales generados contra rEnoHi, mediante ensayos Western-blot y ELISA. Resultados: La inmunización de rEnoHi generó títulos de anticuerpos mayores de 1:80,000; estos anticuerpos mostraron alta especificidad al reconocer a $\mathrm{rEnoHi}$ y a la enolasa nativa de diferentes aislados clínicos de $H$. influenzae (serotipo b y cepas no tipificables). El isotipo de anticuerpos demostró una diferencia significativa de lgG1 con respecto a IgG2a, indicando que $\mathrm{rEnoHi}$ puede desencadenar una respuesta Th1/Th2; sin embargo, se ve polarizada hacia Th2. Discusión: rEnoHi es altamente inmunogénica y puede activar una respuesta inmunitaria tanto humoral 
como celular, es importante considerar esta capacidad inmunogénica en el desarrollo de una vacuna contra $H$. influenzae.

\section{TERCER LUGAR}

Caracterización de enterobacterias resistentes a carbapenémicos obtenidas de pacientes atendidos en un hospital pediátrico de tercer nivel

Aquino-Andrade A, Merida-Vieyra JA, Loyola-Cruz MA, Tapia-Reyes A, De Colsa-Ranero A, Aparicio-Ozores G, Aquino-Andrade A

Instituto Nacional de Pediatría.

Correspondencia: Insurgentes Sur 3700-C, Col. Insurgentes Cuicuilco, 045530, Coyoacán, Ciudad de México. Teléfono: 5510840900, ext.1859.E-mail: aquinoa26@ hotmail.com

Objetivo: Caracterizar a enterobacterias resistentes a carbapenémicos (ERC) aisladas de infecciones de pacientes pediátricos. Tipo y diseño del estudio de estudio: transversal. Material y métodos: Se analizaron 41 aislamientos de ERC colectados de septiembre de 2016 a junio de 2019. Se obtuvieron los perfiles de susceptibilidad. Se realizaron pruebas fenotípicas y genotípicas (amplificación, secuenciación y análisis bioinformático) para la detección de $\beta$-lactamasas. Se estableció la relación clonal mediante PFGE, MLST y filotipificación. Análisis estadístico: estadística descriptiva. Resultados: El perfil de susceptibilidad que mostraron los aislamientos fue ceftazidima $12 \%$, ceftriaxona $15 \%$, cefepima $20 \%$, piperacilina/tazobactam $7 \%$, ciprofloxacino $29 \%$, levofloxacina $54 \%$, amikacina $65 \%$ y gentamicina $71 \%$. Se observó la producción de carbapenemasas en $61 \%$ de las ERC. NDM fue la enzima más frecuente (46\%); seguida de OXA-48 (23\%); VIM (15\%); GES (12\%) y KPC (4\%). En las ERC se encontraron los genes de las $\beta$-lactamasas de espectro extendido (BLEE), CTX-M-15 y SHV-12. Tres aislamientos estrechamente relacionados de K. pneumoniae fueron ST485 y ST45 (2). Ocho aislamientos de E. coli pertenecían al filogrupo $A$, dos al $C$ y uno al $D$; dos aislamientos fueron del complejo clonal de alto riesgo ST10. Los aislamientos de E. cloacae no tuvieron relación clonal. Ética: Aprobado por los comités INP IRB:00008064/00008064), 2018/017. Conclusiones: K. pneumoniae y E. cloacae fueron las principales enterobacterias asociadas con la resistencia a carbapenémicos. $61 \%$ de las ERC fueron productoras de carbapenemasas y de éstas $56 \%$ presentó genes de BLEE. NDM-1 y CTX-M-15 fueron las $\beta$-lactamasas más comunes en ERC. Los aislamientos de K. pneumoniae y $E$. cloacae tuvieron diferente origen clonal. En aislamientos de E. coli sólo se detectó una clona de alto riesgo.

Investigación clínica

\section{PRIMER LUGAR}

Producción residual de radicales libres de oxígeno, riesgo de infecciones y mortalidad en pacientes con enfermedad granulomatosa crónica

León-Lara KX, Medina-Vera I, Yamazaki-Nakashimada MA, Espinosa-Padilla SE, Blancas-Galicia L Instituto Nacional de Pediatría.

Correspondencia: Insurgentes Sur 3700 Letra C, Col. Insurgentes Cuicuilco, 04530, Ciudad de México. Teléfono: 5581711508. E-mail:ximenaleonlara@hotmail.com

Objetivo: Asociar la producción de radicales libres de oxígeno (RLO) con el número y tipo de infecciones graves, microorganismo aislado y mortalidad en pacientes con enfermedad granulomatosa crónica (EGC). Tipo y diseño del estudio: estudio analítico, retrospectivo, observacional. Material y métodos: Se recabaron del expediente clínico los episodios infecciosos graves presentados hasta 2018 de los pacientes con EGC registrados en la Unidad de Investigación en Inmunodeficiencias de 2013 a 2018. La producción residual de RLO se obtuvo calculando el índice de estimulación de los archivos de citometría de flujo con 1,2,3 dihidrorodamina realizada al diagnóstico. La producción de RLO se dicotomizó con base en la mediana en producción moderada y nula. Análisis estadístico: Se calculó el OR (IC 95\%) y $\chi^{2}$ entre la producción de RLO y las variables clínicas. El coeficiente de correlación de Spearman entre la producción de RLO y el número de infecciones. Se realizó un análisis de supervivencia con Kaplan 
Rev Latin Infect Pediatr 2020; 33 (2): 97-106

Meyer entre la producción de RLO dicotomizada. El valor de $p<0.05$ se consideró como significativo. Resultados: Se incluyeron 57 pacientes con EGC, $52(93 \%)$ hombres, la mediana de edad fue cinco años (min. cinco meses-máx. 18 años). La mediana de producción de RLO en neutrófilos fue de 1.4 (mín. 0.66-máx. 7.2). Las infecciones graves más frecuentes fueron neumonía en $38(66 \%)$ y gastroenteritis 27 (47\%). El microorganismo aislado más frecuente fue $S$. aureus $16(28 \%)$, seguido de $S$. marcescens $10(17 \%)$ y Aspergillus spp. ocho (14\%). En 36 (63\%) pacientes se reportó reacción adversa a BCG. Se registraron 14 (24.5\%) muertes, 11 con producción RLO nula. Los pacientes con producción de RLO nula presentaron un OR de 5.4 (IC 95\% 1.5-22) de morir en comparación con los de producción de RLO moderada $(p=0.014)$. No se observó correlación entre el número de infecciones graves, tipo de infección y agente aislado con la producción de RLO. Conclusiones: Los pacientes con EGC y producción residual de RLO nula tienen significativamente mayor riesgo de muerte durante la etapa pediátrica y menos probabilidades de supervivencia a largo plazo que los pacientes con producción residual moderada de RLO. Sin una asociación entre el tipo y número de infecciones con la producción residual de RLO.

\section{SEGUNDO LUGAR}

Prevalencia de virus respiratorios en la población pediátrica del Instituto Nacional de Enfermedades Respiratorias en el periodo 20142019

Mata-Aranda J, Gordillo-Nieto SV, Mújica-Sánchez MA, García-Colín MC, Mireles-Dávalos CD, Becerril-Vargas E, Carreto-Binaghi LE

Instituto Nacional de Enfermedades Respiratorias Ismael Cosío Villegas (INER).

Objetivo: Conocer la epidemiología de virus respiratorios en pacientes pediátricos del INER de 2014 a 2019. Tipo y diseño del estudio: observacional, descriptivo, transversal y retrospectivo. Material y métodos: Se incluyeron pacientes de un mes a 15 años, hospitalizados en el INER por infección de vías respiratorias, a quienes se tomó hisopado nasofaríngeo para PCR multiplex de virus respiratorios de 2014 a 2019. Se analizaron edad y género, fecha de toma de muestra y virus obtenidos. Análisis estadístico: Se utilizó estadística descriptiva para todas las varia- bles. Las correlaciones entre variables se analizaron con prueba de Pearson o Rho Spearman, según el tipo de variable. Se utilizó SPSS v. 21. Resultados: Se estudiaron 2,036 pacientes; la mayoría fueron lactantes (52.7\%) y $56.2 \%$ hombres. Los agentes más frecuentes fueron rinovirus (HRV) (34.8\%), influenza (17.2\%) y virus sincitial respiratorio (RSV) (15.4\%). El tipo de influenza más frecuente fue $A$ H1N1pdm. Se encontró correlación entre la etiología $y$ los grupos de edad $(p=0.000)$. En adolescentes no se identificaron adenovirus (HAdV), bocavirus ( $\mathrm{HBoV}$ ) ni metapneumovirus (hPMV). HRV fue más frecuente en septiembre y febrero; influenza en enero y febrero; RSV y hPMV en octubre y noviembre; parainfluenza en marzo, septiembre y octubre; coronavirus en diciembre y enero; HBoV en noviembre $y$ diciembre; HAdV en marzo y octubre $(p=0.000)$. En 2014 y 2016 HRV e influenza fueron los agentes más frecuentes; en 2015 y 2017, HRV y RSV; en 2018 , HRV y hPMV $(p=0.000)$. No hubo correlación entre el género y el tipo de virus $(p=0.519)$. Ética: Investigación sin riesgo aprobada por el Comité de Ética e Investigación del INER. Conclusiones: Las principales características de los pacientes que presentaron infecciones respiratorias virales fueron: género masculino y edad menor de dos años. HRV, RSV e influenza predominaron en la temporada invernal. Se encontró correlación estadística entre el tipo de virus, la edad y el mes del año.

\section{TERCER LUGAR}

Factores de riesgo asociados a mortalidad por bacterias multidrogo resistentes en pacientes con fiebre y neutropenia de origen oncológico

Minero-Hibert MA, Avilés-Robles M, Moreno-Miranda R, Casillas-Casillas MC, López-Moreno VE

Instituto Nacional de Salud Hospital Infantil de México Federico Gómez.

Correspondencia: Dr. Márquez Núm. 162, Col. Doctores, 06720, Alcaldía Cuauhtémoc, Ciudad de México. Teléfono: 52289917, ext. 2331. E-mail: miguelhibert@gmai.com

Introducción: El uso indiscriminado de antibióticos genera resistencia a dichos tratamientos, induciendo cepas multidrogo resistentes (MDR). Objetivo: Identificar los factores asociados a mortalidad en infecciones producidas por bacterias MDR. Describir incidencia de infecciones MDR y la mortalidad por 
bacterias MDR. Metodología: Se realizó un estudio observacional y analítico de casos y controles anidado en una cohorte prospectiva en el Hospital Infantil de México Federico Gómez del 01.03.15 al 30.09.17 en pacientes pediátricos con evento de fiebre y neutropenia (NF) de origen oncológico. Análisis estadístico: se realizó estadística descriptiva. Resultados: Se registraron 929 eventos de NF, se reportaron aislamientos por MDR en 56 de los mismos, representando $6 \%$ de la población estudiada. $44.4 \%$ de los eventos se observó en el género masculino, la edad media fue de 7.9 años, siendo la población menor de cinco años la de mayor representación con $68.2 \%$. El principal diagnóstico fue LLA en $75 \%$ de los casos. Los agentes aislados que cumplían la definición de MDR, representaron $6.15 \%$, con K. pneumoniae en $22.8 \%$ de los casos. La presencia de mucositis $(p=0.02)$, historia clínica de intubación ( $p=002)$, estancia en la UTIP ( $p<$ $0.001)$ y choque séptico $(p=0.005)$ se asociaron a mortalidad por bacterias MDR. Los pacientes con mortalidad asociada con infección por MDR mostraron una duración mayor de la neutropenia con una media de 8.07 días, en comparación con los pacientes que no fallecieron con una media de 4.93 días (p 0.01). La mortalidad de todos los eventos de NF fue de $1.94 \%$, de los cuales sólo $0.86 \%$ fueron secundarios a bacterias MDR. Conclusiones: Los factores de riesgo asociados con mortalidad fueron: mucositis, historia clínica de intubación, estancia en la UTIP y choque séptico. Las IVU representaron el mayor sitio de infección asociado a infección por MDR en $49.12 \%$. La bacteriemia por un MDR se asocia a mayor riesgo de mortalidad en $25.9 \%$, en comparación con bacteriemia por no MDR en 3.3\%.

\section{TERCER LUGAR}

Identificación rápida de genes de resistencia bacteriana por PCR tiempo real en el laboratorio de microbiología

Juárez-Tapia B, Díaz-Jiménez IV, Tapia-Reyes A Instituto Nacional de Pediatría.
Correspondencia: Insurgentes Sur 3700, Col. Insurgentes Cuicuilco, 04530, Coyoacán, Ciudad de México. Teléfono: 10840900, ext.1517.E-mail:belenjuarezt@gmail.com

Objetivo: Identificación rápida de genes de resistencia presentes en enterobacterias, enterococos y $S$. aureus en el trabajo diario del laboratorio de microbiología. Tipo y diseño de estudio: es un tipo de estudio clínico, prospectivo, observacional, descriptivo. Material y métodos: Se analizarán los estudios a partir de un frasco de hemocultivo positivo, se realizó la identificación del microorganismo por Maldi y se efectuó PCR del gen mecA de $S$. aureus y del gen Van A que le confiere resistencia a vancomicina en los enterococos. A las enterobacterias aisladas que fueron resistentes a carbapenémicos se les realizó PCR para los genes carbapemenasas. Análisis estadístico: Para establecer el tipo de distribución variable cuantitativa y en numéricas continuas con distribución normal se realizará el cálculo de la media y DE y cualitativas, se obtendrá el total y la proporción. En el caso de que las variables a medir no tengan una distribución con tendencia a la normalidad se estimará el valor mínimo y el valor máximo, y se calculará la mediana. Resultados: Cepas de marzo de 2019 a octubre de 2019 aisladas: 102 enterobacterias resistentes a carbapenémicos, de las cuales 35/102 (34\%) fueron Pseudomonas aeruginosa, 18/102 (18\%) Acinetobacter baumannii 11/102 (11\%). Se detectó la presencia de carbapemenasas en 18/112 (16\%) en frecuencia se observó NDM 10/18 (56\%), OXA $485 / 18$ (28\%), IMP 2/18 (11\%), VIM 1/18 (6\%). S. aureus se detectó mecA en 2/32 (6\%). 34 enterococos, de los cuales 20/34 (59\%) fueron E. faecium, 12/34 (35\%) E. faecalis, 1/34 (3\%) E. gallinarum, 1 (3\%) Se encontró 14/20 (70\%) el gen de resistencia vancomicina Van A únicamente en los $E$. faecium. Conclusiones: La identificación rápida de los genes de resistencia es fundamental para dar un tratamiento selectivo en los pacientes y a su vez los pacientes que tienen dichos genes deben tener un aislamiento por el servicio de nosocomiales en los hospitales para evitar diseminación intrahospitalario de gérmenes multirresistentes. 